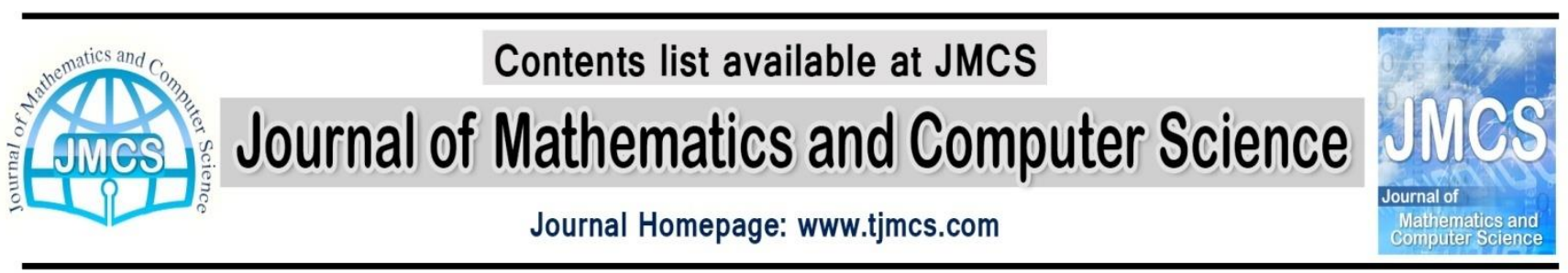

\title{
Introducing a Novel Bivariate Generalized Skew-Symmetric Normal Distribution
}

\author{
Behrouz Fathi-Vajargah \\ Department of Statistics, University of Guilan, Rasht, Iran \\ fathi@guilan.ac.ir \\ Parisa Hasanalipour \\ Department of Statistics, University of Guilan, Rasht, Iran \\ p.hasanalipour116919@gmail.com
}

Article history:

Received April 2013

Accepted May 2013

Available online June 2013

\begin{abstract}
We introduce a generalization of the bivariate generalized skew-symmetric normal distribution [5]. We denote this distribution by $S G N_{n}\left(\lambda_{1}, \lambda_{2}\right)$. We obtain some properties of $S G N_{n}\left(\lambda_{1}, \lambda_{2}\right)$ and derive the moment generating function.
\end{abstract}

Keywords: Generalized-skew-normal distribution, $S G N_{n}\left(\lambda_{1}, \lambda_{2}\right)$, Conditional distribution.

\section{Introduction}

The skew-normal distribution introduced by Azzalini [2]. This density has been studied and generalized by some researchers. For example, Azzalini and Dalla Valle [4], Azzalini and Capitanio [3], Arellano-Valle [1], Jamalizadeh [7], Sharafi and Behbodian [8], Hasanalipour and sharafi [6] and Yadegari [9].

Fathi and Hasanalipour [5] considered a generalization of $S G N_{2}\left(\lambda_{1}, \lambda_{2}\right)$ distribution and they called it the bivariate generalized skew-symmetric normal distribution.

Its probability density function is given by

$$
f\left(x, y ; \lambda_{1}, \lambda_{2}\right)=2 \phi(x) \phi(y) \Phi\left(\frac{\lambda_{1} x y}{1+\lambda_{2}(x y)^{2}}\right), \quad x, y \in R, \quad \lambda_{1} \in R, \quad \lambda_{2} \geq 0
$$

This distribution denoted by $(X, Y) \sim S G N_{2}\left(\lambda_{1}, \lambda_{2}\right)$. In this paper, we introduce a new family of skewnormal distribution which generalizes (1) while preserving most of its properties.

In section 2, we present the definition and some properties of $S G N_{n}\left(\lambda_{1}, \lambda_{2}\right)$ class and section 3, gives some important theorems about conditional distributions of $S G N_{n}\left(\lambda_{1}, \lambda_{2}\right)$. 


\section{A novel bivariate generalized skew-symmetric normal distribution}

In this section, we define the $S G N_{n}\left(\lambda_{1}, \lambda_{2}\right)$ class and obtain some its properties.

2.1. $S G N_{n}\left(\lambda_{1}, \lambda_{2}\right)$

Definition 1. Vector $(X, Y)$ has $S G N_{n}\left(\lambda_{1}, \lambda_{2}\right)$ distribution if and only if for every $n \geq 1$ it has the following density

$$
f_{n}\left(x, y ; \lambda_{1}, \lambda_{2}\right)=c_{n}\left(\lambda_{1}, \lambda_{2}\right) \phi(x) \phi(y) \Phi^{n}\left(\frac{\lambda_{1} x y}{\sqrt{1+\lambda_{2}(x y)^{2}}}\right), \quad x, y \in R,
$$

where $\lambda_{1} \in R$ and $\lambda_{2} \geq 0$. The coefficient $c_{n}\left(\lambda_{1}, \lambda_{2}\right)$, which is a function of $n$ and the parameters $\lambda_{1}, \lambda_{2}$ is given by

$$
c_{n}\left(\lambda_{1}, \lambda_{2}\right)=\frac{1}{\int_{-\infty}^{\infty} \int_{-\infty}^{\infty} \phi(x) \phi(y) \Phi^{n}\left(\frac{\lambda_{1} x y}{\sqrt{1+\lambda_{2}(x y)^{2}}}\right) d x d y},
$$

with this properties:

1. $\lim _{\lambda_{1} \rightarrow \infty} c_{n}\left(\lambda_{1}, \lambda_{2}\right)=4$ for all $\lambda_{2} \geq 0$

2. $c_{n}\left(-\lambda_{1}, \lambda_{2}\right)=c_{n}\left(\lambda_{1}, \lambda_{2}\right)$

We denote this by $(X, Y) \sim S G N_{n}\left(\lambda_{1}, \lambda_{2}\right)$.

\subsection{Some simple properties of $S G N_{n}\left(\lambda_{1}, \lambda_{2}\right)$}

We now present some properties of this novel distribution.

1. $S G N_{n}\left(0, \lambda_{2}\right)=\phi(x) \phi(y)$, for all $\lambda_{2} \geq 0$.

2. $S G N_{1}\left(\lambda_{1}, 0\right)=2 \phi(x) \phi(y) \Phi\left(\lambda_{1} x y\right)$.

3. $X \mid\{Y=y\}=c_{n}\left(\lambda_{1} y\right) \phi(x) \Phi^{n}\left(\frac{\lambda_{1} x y}{\sqrt{1+\lambda_{2}(x y)^{2}}}\right) \sim \operatorname{GBSN}_{n}\left(\lambda_{1} y\right)[6]$.

4. $Y \mid\{X=x\}=c_{n}\left(\lambda_{1} x\right) \phi(y) \Phi^{n}\left(\frac{\lambda_{1} x y}{\sqrt{1+\lambda_{2}(x y)^{2}}}\right) \sim \operatorname{GBSN}_{n}\left(\lambda_{1} x\right)[6]$.

5. If $(X, Y) \sim S G N_{n}\left(\lambda_{1}, \lambda_{2}\right)$, then $(-X, Y) \sim S G N_{n}\left(-\lambda_{1}, \lambda_{2}\right),(X,-Y) \sim S G N_{n}\left(-\lambda_{1}, \lambda_{2}\right)$ and $(-X,-Y) \sim S G N_{n}\left(\lambda_{1}, \lambda_{2}\right)[1]$ 
6. $\lim _{\lambda_{1} \rightarrow \infty} f_{n}\left(x, y ; \lambda_{1}, \lambda_{2}\right)=4 \phi(x) \phi(y) I_{\{x>0, y>0\}}$.

7. $\lim _{\lambda_{1} \rightarrow-\infty} f_{n}\left(x, y ; \lambda_{1}, \lambda_{2}\right)=4 \phi(x) \phi(y) I_{\{x<0, y<0\}}$.

8. $\lim _{\lambda_{1} \rightarrow \infty}\left\{f_{X \mid Y}\left(x, y ; \lambda_{1}, \lambda_{2}\right)+f_{X \mid Y}\left(x, y ;-\lambda_{1}, \lambda_{2}\right)\right\}=2 \phi(x)$.

9. $\lim _{\lambda_{1} \rightarrow \infty}\left\{f_{Y \mid X}\left(x, y ; \lambda_{1}, \lambda_{2}\right)+f_{Y \mid X}\left(x, y ;-\lambda_{1}, \lambda_{2}\right)\right\}=2 \phi(y)$.

\section{Some theorems about conditional distributions of $S G N_{n}\left(\lambda_{1}, \lambda_{2}\right)$}

Theorem 1. If $X, Y, Z_{1}, \ldots, Z_{n}$ are i.i.d. $N(0,1)$ distribution then we have:

$$
(X, Y) \mid\left\{Z_{(n)} \leq \frac{\lambda_{1} X Y}{\sqrt{1+\lambda_{2}(X Y)^{2}}}\right\} \sim S G N_{n}\left(\lambda_{1}, \lambda_{2}\right)
$$

Where $Z_{(n)}=\max \left\{Z_{1}, \ldots, Z_{n}\right\}$.

Proof: Suppose $A=\left(Z_{(n)} \leq \frac{\lambda_{1} X Y}{\sqrt{1+\lambda_{2}(X Y)^{2}}}\right)$. Then, we write

$$
\begin{aligned}
f_{(X, Y) \mid A}(x, y \mid A)= & \frac{P(A \mid X=x, Y=y) f(x, y)}{P(A)} \\
= & \frac{P\left(Z_{(n)} \leq \frac{\lambda_{1} X Y}{\sqrt{1+\lambda_{2}(X Y)^{2}}} \mid X=x, Y=y\right) \phi(x) \phi(y)}{P\left(Z_{(n)} \leq \frac{\lambda_{1} X Y}{\sqrt{1+\lambda_{2}(X Y)^{2}}}\right)} \\
= & \frac{P\left(Z_{1} \leq \frac{\lambda_{1} x y}{\sqrt{1+\lambda_{2}(x y)^{2}}}, \ldots, Z_{n} \leq \frac{\lambda_{1} x y}{\sqrt{1+\lambda_{2}(x y)^{2}}}\right) \phi(x) \phi(y)}{P\left(Z_{1} \leq \frac{\lambda_{1} X Y}{\sqrt{1+\lambda_{2}(X Y)^{2}}}, \ldots, Z_{n} \leq \frac{\lambda_{1} X Y}{\sqrt{1+\lambda_{2}(X Y)^{2}}}\right)} \\
= & c_{n}\left(\lambda_{1}, \lambda_{2}\right) \phi(x) \phi(y) \Phi^{n}\left(\frac{\lambda_{1} x y}{\sqrt{1+\lambda_{2}(x y)^{2}}}\right)
\end{aligned}
$$

For random number generation, it is more efficient to use single variant of this result, namely to put 


$$
Z=\left(Z_{1}, Z_{2}\right)=\left\{\begin{array}{lc}
(X, Y) & Z_{(n)} \leq \frac{\lambda_{1} X Y}{\sqrt{1+\lambda_{2}(X Y)^{2}}} \\
(-X,-Y) & Z_{(n)}>\frac{\lambda_{1} X Y}{\sqrt{1+\lambda_{2}(X Y)^{2}}}
\end{array}\right.
$$

This make an important point for $S G N_{n}\left(\lambda_{1}, \lambda_{2}\right)$ distribution, comparing with acceptance-rejection method simulation of independent normal distribution.

Theorem 2. If $(X, Y) \sim S G N_{n}\left(\lambda_{1}, \lambda_{2}\right)$, then $\left(X^{2} \mid Y\right) \stackrel{L}{\rightarrow} \chi_{(1)}^{2}$ as $\lambda_{1} \rightarrow \infty$, where $\chi_{(1)}^{2}$ shows chi-square random variable with one degree of freedom.

Proof: Let $\left(X^{2} \mid Y\right)=Z$. The density of $Z$ is

$$
\begin{aligned}
f_{Z}\left(z, y ; \lambda_{1}, \lambda_{2}\right) & =\frac{1}{\sqrt{z}} \frac{1}{\sqrt{2 \pi}} e^{-\frac{z}{2}} c_{n}\left(\lambda_{1} y\right)\left[\frac{\Phi^{n}\left(\frac{\lambda_{1} \sqrt{z} y}{\sqrt{1+\lambda_{2} z y^{2}}}\right)+\Phi^{n}\left(\frac{-\lambda_{1} \sqrt{z} y}{\sqrt{1+\lambda_{2} z y^{2}}}\right)}{2}\right] \\
& =f_{\chi_{(1)}^{2}}(z)\left[a_{n}\left(z, y ; \lambda_{1}, \lambda_{2}\right)\right] ; \quad z>0
\end{aligned}
$$

with

$$
a_{n}\left(z, y ; \lambda_{1}, \lambda_{2}\right)=c_{n}\left(\lambda_{1} y\right)\left[\frac{\Phi^{n}\left(\frac{\lambda_{1} \sqrt{z} y}{\sqrt{1+\lambda_{2} z y^{2}}}\right)+\Phi^{n}\left(\frac{-\lambda_{1} \sqrt{z} y}{\sqrt{1+\lambda_{2} z y^{2}}}\right)}{2}\right]
$$

Since $c_{n}\left(\lambda_{1} y\right) \rightarrow 2$ as $\lambda_{1} \rightarrow \infty$, we conclude that $a_{n}\left(z, y ; \lambda_{1}, \lambda_{2}\right) \rightarrow 1$, as $\lambda_{1} \rightarrow \infty$. Therefore, the density $f_{Z}\left(z, y ; \lambda_{1}, \lambda_{2}\right)$ converges to the distribution of $\chi_{(1)}^{2}$, as $\lambda_{1} \rightarrow \infty$. Hence, the distribution of $Z$ converges to the distribution of $\chi_{(1)}^{2}$, i.e. $Z=\left(X^{2} \mid Y\right) \stackrel{L}{\longrightarrow} \chi_{(1)}^{2}$.

Theorem 3. If $(X, Y) \sim S G N_{n}\left(\lambda_{1}, \lambda_{2}\right)$ and $Z \sim N(0,1)$, then $\frac{|X|}{Y}$ and $|Z|$ are identically distributed, i.e, $\lim _{\lambda_{1} \rightarrow \infty} \frac{|X|}{Y} \stackrel{D}{\rightarrow}|Z| \sim H N(0,1)$, where $H N(0,1)$ denotes the standard half-normal distribution.

Proof: We know that $|Z|$ has density $2 \phi(z) I_{\{z>0\}}$. The density $W=\frac{|X|}{Y}$ is 


$$
\begin{aligned}
f_{W}(w) & =f_{X|Y|}(w)+f_{X|Y|}(-w) \\
& =c_{n}\left(\lambda_{1} y\right) \phi(w) \Phi^{n}\left(\frac{\lambda_{1} w y}{\sqrt{1+\lambda_{2}(w y)^{2}}}\right)+c_{n}\left(\lambda_{1} y\right) \phi(-w) \Phi^{n}\left(\frac{-\lambda_{1} w y}{\sqrt{1+\lambda_{2}(w y)^{2}}}\right) \\
& =c_{n}\left(\lambda_{1} y\right) \phi(w)\left[\Phi^{n}\left(\frac{\lambda_{1} w y}{\sqrt{1+\lambda_{2}(w y)^{2}}}\right)+\Phi^{n}\left(\frac{-\lambda_{1} w y}{\sqrt{1+\lambda_{2}(w y)^{2}}}\right)\right] \\
& =\phi(w)\left[b_{n}\left(w, y ; \lambda_{1}, \lambda_{2}\right)\right]
\end{aligned}
$$

Now, we can show that $b_{n}\left(w, y ; \lambda_{1}, \lambda_{2}\right) \rightarrow 2$ as $\lambda_{1} \rightarrow \infty$, then $\lim _{\lambda_{1} \rightarrow \infty} W=2 \phi(w)$ for $w>0$ and we have $\lim _{\lambda_{1} \rightarrow \infty} \frac{|X|}{Y} \stackrel{D}{\rightarrow}|Z|$

Theorem 4. The moment generating function $(X, Y) \sim S G N_{n}\left(\lambda_{1}, \lambda_{2}\right)$ is

$$
M_{X, Y}\left(t_{1}, t_{2}\right)=c_{n}\left(\lambda_{1}, \lambda_{2}\right) e^{\frac{t_{1}^{2}+t_{2}^{2}}{2}} E\left\{E\left\{\Phi^{n}\left(\frac{\lambda_{1} W K}{\sqrt{1+\lambda_{2}(W K)^{2}}}\right)\right\}\right\}
$$

where $W \sim N\left(t_{1}, 1\right), K \sim N\left(t_{2}, 1\right)$.

Proof:

$$
\begin{aligned}
M_{X, Y}\left(t_{1}, t_{2}\right) & =E\left(e^{t_{1} X+t_{2} Y}\right) \\
& =\int_{-\infty}^{\infty} \int_{-\infty}^{\infty} c_{n}\left(\lambda_{1}, \lambda_{2}\right) e^{t_{1} x+t_{2} y} \phi(x) \phi(y) \Phi^{n}\left(\frac{\lambda_{1} x y}{\sqrt{1+\lambda_{2}(x y)^{2}}}\right) d x d y \\
& =c_{n}\left(\lambda_{1}, \lambda_{2}\right) e^{\frac{t_{1}^{2}+t_{2}^{2}}{2}} E\left\{E\left\{\Phi^{n}\left(\frac{\lambda_{1} W K}{\sqrt{1+\lambda_{2}(W K)^{2}}}\right)\right\}\right\} .
\end{aligned}
$$

\section{References}

[1] R. B. Arellano-Valle, H. W. Gomez and F. A. Quintana, A new class of skew-normal distribution. Commun. Stat Theory Methods, 33(7), 1465-1480 (2004).

[2] A. Azzalini, A class of distributions which includes the normal ones. Scand J Stat. 12,171-178 (1985). [3] A. Azzalini and A. Capitanio, Statistical application of the multivariate skew-normal distribution. J. R. Statist. Soc B, 61,579-602 (1999).

[4] A. Azzalini and A. Dalla-Valle, The multivariate skew-normal distribution. Biometrika 83, 715-726 (1996).

[5] B. Fathi and P. Hasanalipour, Simulation and theory of bivariate generalized skew-symmetric normal distribution. Under review (2012).

[6] P. Hasanalipour and M. Sharafi, A new generalized Balakrishnan skew-normal distribution. Statistical Papers, 53, 219-228 (2010). 
[7] A. Jamalizadeh, J. Behboodian and N. Balakrishnan, A two-parameter generalized skew-normal distribution. Statistics and Probability Letters. 78, 1722-1726 (2008).

[8] M. Sharafi, J. Behboodian, The Balakrishnan skew-normal density. Statistical Papers. 49, 769-778 (2008).

[9] I. Yadegari, A. Gerami and M.J Khaledi, A generalization of the Balakrishnan skew-normal distribution. Statistics and Probability Letters. 78, 1165-1167 (2008). 